\title{
Singular Integrals and Related Topics
}


This page intentionally left blank 
Singular Integrals and Related Topics

\title{
Shanzhen Lu Yong Ding
}

Beijing Normal University, China

\section{Dunyan Yan}

\author{
Academia Sinica, China
}

\section{|fo world Scientific}


Published by

World Scientific Publishing Co. Pte. Ltd.

5 Toh Tuck Link, Singapore 596224

USA office: 27 Warren Street, Suite 401-402, Hackensack, NJ 07601

UK office: 57 Shelton Street, Covent Garden, London WC2H 9HE

\section{British Library Cataloguing-in-Publication Data}

A catalogue record for this book is available from the British Library.

\section{SINGULAR INTEGRALS AND RELATED TOPICS}

Copyright @ 2007 by World Scientific Publishing Co. Pte. Ltd.

All rights reserved. This book, or parts thereof, may not be reproduced in any form or by any means, electronic or mechanical, including photocopying, recording or any information storage and retrieval system now known or to be invented, without written permission from the Publisher.

For photocopying of material in this volume, please pay a copying fee through the Copyright Clearance Center, Inc., 222 Rosewood Drive, Danvers, MA 01923, USA. In this case permission to photocopy is not required from the publisher.

ISBN-13 978-981-270-623-2

ISBN-10 981-270-623-2

Printed in Singapore. 


\section{Preface}

It is well-known that singular integrals is continuously regarded as a central role in harmonic analysis. There are many nice books related to singular integrals. In this book, there are at least two sides which differ from the other books. One of them is to establish more perfect theory of singular integrals. It includes not only the case of smooth kernels, but also the case of rough kernels. In the same way, we deal with some related operators, such as fractional integral operators and Littlewood-Paley operators. The other is to introduce more new theory on some oscillatory singular integrals with polynomial phases. This book is mainly provided to graduate students in analysis field. However, it is also beneficial to researchers in mathematics.

This book consists of five chapters. Let us now illustrate the choice of material in each chapter. Chapter 1 is devoted to the theory of the Hardy-Littlewood maximal operator as the basis of singular integrals and other related operators. It also includes the basic theory of the $A_{p}$ weights. Chapter 2 is related to the theory of singular integrals. Since the theory of singular integrals with Calderón-Zygmund kernel has been introduced in many books, we will pay more attention to the singular integrals with homogeneous kernels. Specially, we will introduce more perfect theory of singular integrals with rough kernels, for instance the $L^{p}$ boundedness of singular integrals with kernels in certain Hardy space on the unit sphere will be fully proved. In addition, the weighted $L^{p}$ boundedness of singular integrals with rough kernels and their commutators will be also established. Chapter 3 is devoted to fractional integrals. In the same way, we will pay more attention to the case of rough kernels. It includes not only the $A(p, q)$ weight theory of fractional integrals with rough kernels, but also the theory of its commutators. Chapter 4 is to introduce a class of oscillatory singular integrals with polynomial phases. Note that this oscillatory singular integral is neither a Calderón-Zygmund operator nor a convolution operator. However there exists certain link between this oscillatory singular integral and the corre- 
sponding singular integral. Therefore, the criterion on the $L^{p}$ boundedness of oscillatory singular integrals will become a crucial role in this chapter. It will discover an equivalent relation between the $L^{p}$ boundedness of the oscillatory singular integral and that of the corresponding truncated singular integral. Chapter 5 is related to the Littlewood-Paley theory. In this chapter, we will establish two kinds of the weakest conditions on the kernel for the $L^{p}$ boundedness of Marcinkiewicz integral operator with rough kernel. Finally, it is worth pointing out that as space is limited, the theory of singular integrals and related operators in this book is only worked on the Lebesgue spaces although there are many good results on other spaces such as Hardy spaces and BMO space.

It should be pointed out that many results in the later three chapters of this book reflect the research accomplishment by the authors of this book and their cooperators. We would like to acknowledge to Jiecheng Chen, Dashan Fan, Yongsheng Han, Yingsheng Jiang, Chin-Cheng Lin, Guozhen Lu, Yibiao Pan, Fernando Soria and Kozo Yabuta for their effective cooperates in the study of singular integrals. On this occasion, the authors deeply cherish the memory of Minde Cheng and Yongsheng Sun for their constant encourage. The first named author of this book, Shanzhen Lu, would like to express his thanks to his former students Wengu Chen, Yong Ding, Zunwei $\mathrm{Fu}$, Yiqing Gui, Guoen Hu, Junfeng Li, Guoquan Li, Xiaochun Li, Yan Lin, Heping Liu, Mingju Liu, Zhixin Liu, Zongguang Liu, Bolin Ma, Huixia Mo, Lin Tang, Shuangping Tao, Huoxiong Wu, Qiang Wu, Xia Xia, Jingshi Xu, Qingying Xue, Dunyan Yan, Dachun Yang, Pu Zhang, and Yan Zhang for their cooperations and contributions to the study of harmonic analysis during the joint working period. Finally, Shanzhen Lu would like to express his deep gratitude to Guido Weiss for his constant encourage and help.

Shanzhen Lu

Yong Ding

Dunyan Yan

December, 2006 


\section{Contents}

Preface $\quad$ v

1 Hardy-Littlewood Maximal Operator 1

1.1 Hardy-Littlewood maximal operator . . . . . . . . . . . 1

1.2 Calderón-Zygmund decomposition . . . . . . . . . . 9

1.3 Marcinkiewicz interpolation theorem . . . . . . . . . 15

1.4 Weighted norm inequalities . . . . . . . . . . . . . . 21

1.5 Notes and references . . . . . . . . . . . . . 35

2 Singular Integral Operators 37

2.1 Calderón-Zygmund singular integral operators . . . . . . . . 40

2.2 Singular integral operators with homogeneous kernels . . . . . 78

2.3 Singular integral operators with rough kernels . . . . . . . . . 92

2.4 Commutators of singular integral operators . . . . . . . . . 119

2.5 Notes and references . . . . . . . . . . . . . . . 131

3 Fractional Integral Operators 133

3.1 Riesz potential . . . . . . . . . . . . . . . . . 134

3.2 Weighted boundedness of Riesz potential . . . . . . . . . 137

3.3 Fractional integral operator with homogeneous kernels . . . . 144

3.4 Weighted boundedness of $T_{\Omega, \alpha} \ldots \ldots \ldots \ldots$. . . . . . . . . . . . . 148

3.5 Commutators of Riesz potential . . . . . . . . . . . 158

3.6 Commutators of fractional integrals with rough kernels . . . . 162

3.7 Notes and references . . . . . . . . . . . . . 166

4 Oscillatory Singular Integrals 169

4.1 Oscillatory singular integrals with homogeneous smooth kernels169

4.2 Oscillatory singular integrals with rough kernels . . . . . . 186

4.3 Oscillatory singular integrals with standard kernels . . . . 197

4.4 Multilinear oscillatory singular integrals with rough kernels . 202 
4.5 Multilinear oscillatory singular integrals with standard kernels 213

4.6 Notes and references . . . . . . . . . . . . . . . 230

5 Littlewood-Paley Operator 233

5.1 Littlewood-Paley g function . . . . . . . . . . . . . . . . 234

5.2 Weighted Littlewood-Paley theory . . . . . . . . . . . . . 241

5.3 Littlewood-Paley g function with rough kernel . . . . . . . . 247

5.4 Notes and references . . . . . . . . . . . . . . . . . 259

$\begin{array}{ll}\text { Bibliography } & 261\end{array}$

$\begin{array}{ll}\text { Index } & 271\end{array}$ 